\title{
Effectiveness of extracorphorus shock wave therapy in musculoskeletal pathologies
}

\section{Introduction}

The Extracorporeal Shock Wave Therapy (ESWT) method was discovered in the 1980s and, initially, its purpose was to destroy kidney stones (urological lithotripsy). ${ }^{1}$ In 1998, it arrived in Brazil with this same design, being later adapted for application in orthopedic injuries. Such adaptation was based on the use of a new technology that allowed to graduate the force and the depth with which the shock waves penetrate the human body. ESWT is able to promote a mechanical stimulus when it reaches the injured site, inducing a series of biological effects, such as: increased local blood microcirculation, increased production of prostaglandins related to the tissue repair process and increased nitric oxide concentration local with pain relief. ${ }^{2}$ Currently, it has been abundantly adopted in the regenerative treatment of musculoskeletal diseases, as it has peculiarities that facilitate the regeneration of damaged tendons and bones, in addition to reducing the pain when used in pathologies such as tendinopathies, plantar fasciitis, epicondylitis lateral elbow, late union of long bone fractures and avascular necrosis of the femoral head. ${ }^{1}$ Its release properties of nitric oxide (NO) and substance $\mathrm{P}$ at the application site, as well as the inhibition of the COX II enzyme, provide an anti-inflammatory effect. The increase in angiogenesis (neovascularization) has also been proven after experimental microscopic analysis on tissues previously submitted to treatment. ${ }^{3}$

It is subdivided into two types: focal and radial. The focal points have great penetrating power in the tissues $(10 \mathrm{~cm})$ and impact force $(0.28-0.6 \mathrm{mj} / \mathrm{mm} 2)$, providing mechanical and biological effects, such as stimulation of tissue neovascularization and destruction of fibrosis. Radials are pneumatic waves produced by air compressions, which are transmitted radially with shorter penetration $(3 \mathrm{~cm})$, less impact $(0.02-0.08 \mathrm{~mJ} / \mathrm{mm} 2)$ and limited biological effect. They are effective in more superficial musculoskeletal conditions. Nevertheless, they cause disintegration of calcifications and fibrosis and increase local blood circulation. ${ }^{4}$

Contraindications to ESWT are: pregnancy, infection in the area to be treated, neoplasms, children under 18 years of age, epiphysis in adolescents, ribs, bones vertebral and skull disorders, coagulation disorders and use of anticoagulant medication. ${ }^{5}$ Its side effects are repeatedly transient and are associated with the energy density of the wave, which usually occur when it is higher. Nausea / dizziness, migraines, pain during treatment, petechiae and redness of the skin can be mentioned. Other side effects include error in the direction of the waves, which can result in nerve or vascular damage. ${ }^{6}$

Studies have been developed with the purpose of investigating the action of ESWT in different human tissues, verifying the intensity, depth, interval between applications, side effects and effectiveness. Treatment is indicated for patients with chronic pain for at least three months and who have not obtained results with other conservative treatments, such as physical therapy, medications, infiltrations and orthoses. The procedure is not adopted in cases of acute pathologies, and its protocol may vary according to the type of wave, and the focal ones, as they are more intense and deeper than the radias, require fewer sessions. ${ }^{2}$
Volume 5 Issue 3 - 2020

\author{
Vespoli Carolina Bageto,' Damázio Laila \\ Cristina Moreira ${ }^{2}$ \\ 'Graduate Student of Trauma-Orthopedic Physiotherapy at \\ President Antônio Carlos University, Brazil \\ ${ }^{2}$ Teacher of the Post-Graduation Course in Traumato- \\ Orthopedic Physiotherapy at the President Antonio Carlos \\ University (UNIPAC-Barbacena), Teacher of Anatomy, \\ Neuroanatomy and Physiology at the University Center \\ President Tancredo de Almeida Neves-UNIPTAN, Teacher of \\ Anatomy and Neuroanatomy at the Federal University of São \\ João del-Rei, UFSJ-Department of Medicine, Brazil
}

\begin{abstract}
Correspondence: Damázio Laila Cristina Moreira, Teacher of the Post-Graduation Course in Traumato-Orthopedic Physiotherapy at the President Antonio Carlos University (UNIPAC-Barbacena), Teacher of Anatomy, Neuroanatomy and Physiology at the University Center President Tancredo de Almeida Neves-UNIPTAN, Teacher of Anatomy and Neuroanatomy at the Federal University of São João del-Rei, UFSJ-Department of Medicine, Brazil, Email lailadamazio@ufsj.edu.br
\end{abstract}

Received: April 16, 2020 | Published: May 07, 2020

This study aims to investigate the effectiveness of ESWT in diseases of the musculoskeletal system, thus being able to assist professionals in the field of orthopedics to understand the effects of this technique and its use in treatments where there has been no success with conservative methods.

\section{Methodology}

This is a systematic review of the literature, where a search was conducted on the following sites: Pubmed and Scielo. Articles covering the period from 2004 to 2017 were selected, using the following keywords: musculoskeletal diseases, high-energy shock waves and conservative treatment. In all, 317 articles were found; titles, abstracts and full texts were read, including only those that fit the purpose of the study, that is, experimental studies and/or systematic reviews involving ESWT in the musculoskeletal diseases most found in the literature; and those who did not clearly address the referred subject or who dispersed it, such as, for example, titles addressing ESWT in urological lithotripsy, were excluded. Publications in Portuguese, English and Spanish were covered. A total of 15 articles were used, including randomized, prospective, comparative clinical studies and systematic reviews.

\section{Results and discussion}

In the study by Androsoni, ${ }^{3}$ it was revealed that there is efficacy in the use of ESWT in plantar fasciitis. Thirty patients (36 feet) resistant to other conservative treatments were recruited. These were submitted to a weekly session of ESWT for four consecutive weeks, using the radial generator. They were also instructed to perform active stretching 
of the posterior chain. The result was a statistically significant decrease in the thickness of the plantar fascia of patients undergoing therapy ( $p$ $<0.001$ ), along with decreased pain and recovery of functionality.

Strike $^{7}$ also carried out a study to determine the success of ESWT in plantar fasciitis. It is a comparative study between two types of protocol: the first consists of the application of ultrasound and kinesiotherapy, with stretching of the posterior muscular chain (hamstrings and sural triceps) and strengthening of the anterior tibial performed by the physiotherapist, together with instruction for active stretching. gastrocnemius and plantar fascia to be performed at home. The second is based on the radial ESWT associated with instructions for active stretching of the gastrocnemius and plantar fascia to be performed at home. In this study, 32 patients were treated, 16 of whom followed the first protocol (10 sessions) and the other 16 followed the second (three sessions). The result obtained was that both protocols were effective in reducing pain and functional disability for at least three months after treatment.

A third study was carried out involving plantar fasciitis. Malliaropoulos et al. ${ }^{8}$ aimed to report the recurrence rate of plantar fasciitis after treatment with ESWT. 68 individuals were analyzed with a minimum pain duration of three months. The number of sessions and the type of generator varied between the subjects, depending on the healing process, the severity and insistence of the symptoms. All of them were treated for a minimum of four to six sessions, with one session being held weekly. The effects of ESWT were assessed in all patients through organized follow-up at one month, three months and one year after treatment. Success rates were calculated at $19 \%$ (15 heels) one month after treatment, 70\% (54 heels) at three months after treatment and 98\% (73 heels) after one year of treatment. The recurrence rate was only $8 \%$ (5 patients) after one year.

Moya $^{9}$ described a study whose objective was to evaluate the result of ESWT in the treatment of calcification of any rotator cuff tendon with radiographic diagnosis. The focal generator was used in a sample of 44 shoulders from 43 patients who were unsuccessful with previous treatments, with symptoms ranging from six months to three and a half years. The treatment was carried out for two weeks, but the number of sessions was not specified. According to the VAS (visual analog scale), the decrease in pain and recovery from daily life activity (DLAs) was considerable $(\mathrm{p}<0.0001)$. After radiographic analysis, calcification disappeared completely in 20 patients $(45.5 \%)$, decreased its size or density in 13 cases $(29.5 \%)$ and did not respond in 11 cases $(25 \%)$. Lee ${ }^{10}$ explored the same subject in a systematic review, dealing with the effectiveness of ESWT in calcareous tendonitis of the rotator cuff, promoting pain reduction and improving shoulder function in the medium term. However, this review noted limitations in the evidence due to the absence of a follow-up period, preventing the conclusion about its effect.

Ioppolo et al. ${ }^{11}$ also showed in their systematic review the functional improvement and the reduction of pain in patients with calcareous tendonitis of the shoulder, in addition to determining the rate of disappearance of the calcifications after six months of therapy. In the study by Huisstede et al. ${ }^{12}$ no positive results were found for non-calcified shoulder tendonitis.

From another perspective, Almeida et al..$^{13}$ assessed in his research the effectiveness of physical therapy treatments in the treatment of lateral epicondylitis. In his survey, despite the limitations of the articles, it was emphasized that the best treatment option is the combination of therapeutic modalities and the isolated use of ESWT was not successful in treating this pathology.

Qingyu et al. ${ }^{14}$ carried out an updated review in order to elucidate the success of ESWT in the treatment of osteonecrosis of the femoral head, proving that the therapy provides improvement of motor function and pain relief, as bone marrow edema is significantly reduced. However, the necrotic bone or the collapse of the femoral head has not been reversed. It is concluded, then, that the technique can delay or block osteonecrosis of the femoral head and consequently reduce the demand for surgical interventions.

Finally, Schmitz et al. ${ }^{15}$ prepared a review comparing ESWT with other conservative treatments for tendons and other musculoskeletal pathologies, using radial and focal waves depending on the case. ESWT has been proven to be effective and should be considered by physicians and physiotherapists as a treatment option for pathologies such as plantar fasciitis, supraspinatus tendinopathies, calcareous rotator cuff tendonitis, Achilles tendonitis and lateral epicondylitis.

\section{Conclusion}

It is concluded that ESWT is effective in the treatment of pathologies of the musculoskeletal system, improving the quality of life of patients with these injuries.

\section{Acknowledgments}

None.

\section{Conflicts of interest}

The authors declare have no conflict of interest about the publication of this paper.

\section{References}

1. Cho YS, JOO SY, Cui H, et al. Effect of extracorporeal shock wave therapy on scar pain in burn patients: A prospective, randomized, single-blind, placebo-controlled study. Medicine. 2016;95(32):e4575.

2. Kertzmana P, Lenzab M, Pedrinelli A, et al. Shockwave treatment for musculoskeletal diseases and bone consolidation: qualitative analysis of the literature. Revista Brasileira de Ortopedia. 2015;50(1):3-8.

3. Androsoni R, Netto AA, Macedo RR, et al. Treatment of chronic plantar fasciitis with extra corporeal shock wave therapy: Ultrasonographic morphological aspect and functional evaluation. Revista Brasileira de Ortopedia. 2013;48(6):538-544.

4. Grecco MV, Brech GC, Greve JMD. One-year treatment follow-up of plantar fasciitis: radial shockwaves vs. conventional physiotherapy. Clinicas vol. 2013;68(8):1089-1095.

5. Hung SN, Veitía CW, Sánchez MM, et al. Ondas de choque em población deportiva y no deportiva: resultados preliminares. Acta ortop. $2015 ; 29(5)$.

6. Harniman E, Carette S, Kennedy C, et al. Extracorporeal shock wave therapy for calcific and noncalcific tendonitis of the rotator cuff: a systematic review. Journal of Hand Therapy. Jun. 2004;17(2):132-151.

7. Greve JMD, Grecco MV, Silva PRS. Comparison of radial shockwaves and conventional physiotherapy for treating plantar fasciitis. Clínicas. 2009;64(2):97-103.

8. Malliarpoulos N, Crate G, Meke M, et al. Success and recurrence rate after radial extracorporeal shock wave therapy for plantar fasciopathy: a retrospective study. Biomed Res Int. 2016:9415827. 
9. Moya D, Patiño O. Results of focal shock wave therapy in rotator cuff calcifications. Rev Asoc Argent Ortop Traumatol. 2012;77(4).

10. Lee SY, Cheng B, Somers KG. The midterm effectiveness of extracorporeal shockwave therapy in the management of chronic calcific shoulder tendinitis. J Shoulder Elbow Surg. 2011;20(5):845854 .

11. Ioppolo F, Tattoli M, Sante LD, et al. Clinical improvement and resorption of calcifications in calcific tendinitis of the shoulder after shock wave therapy at 6 months' follow-up: a systematic review and meta-analysis. Archives of Physical Medicine and Rehabilitation. 2013;94(9):1699-1706.

12. Huisstede BMA, Gebremariam L, Sande RVD, et al. Evidence for effectiveness of extracorporal shock-wave therapy (ESWT) to treat calcific and non-calcific rotator cuff tendinosis - A systematic review. Manual Therapy. 2011;16(5):419-433.
13. Almeida MO, Saragiotto BT, Yamato TP, et al. Tratamento fisioterapêutico para epicondilite lateral: uma revisão sistemática. Fisioter. 2013;26(4).

14. Qingyu Z, Lihua L, Wei S, et al. Extracorporeal shockwave therapy in osteonecrosis of femoral head: A systematic review of now available clinical evidences. Medicine. 2017;96(4):e5897.

15. Schmitz C, Császár NBM, Milz S, et al. Efficacy and safety of extracorporeal shock wave therapy for orthopedic conditions: a systematic review on studies listed in the PEDro database. Br Med Bull. 2015;116:115-38. 